\title{
NLTE $\mathrm{NH}_{3}(\mathrm{~J}, \mathrm{~K})=(1,1)$ observations towards southern hemisphere compact regions
}

\author{
Alexandre Roman Lopes, Zulema Abraham, Anderson Caproni, Jacques \\ R. D. Lépine \\ Instituto Astronômico e Geofísico, Universidade de São Paulo, Brazil \\ Jose W. S. Vilas-Boas \\ Instituto de Pesquisas Espaciais, Brazil
}

\begin{abstract}
We present $\mathrm{NH}_{3}(\mathrm{~J}, \mathrm{~K})=(1,1)$ observations toward $\mathrm{CS}(2,1)$ sources in the southern hemisphere, obtained with the Itapetinga Radio Telescope ${ }^{1}$, that exhibit departure from LTE conditions. The mechanism of selective trapping in the hyperfine transitions of $\mathrm{NH}_{3}(\mathrm{~J}, \mathrm{~K})=(2,1)-(1,1)$ is invoked to explain the non-thermal population in the $\mathrm{NH}_{3}(\mathrm{~J}, \mathrm{~K})=(1,1)$ hyperfine states. This effect is relevant only when the width of the hyperfine lines lie between 0.3 and $0.6 \mathrm{~km} \mathrm{~s}^{-1}$. Due to this restriction, the assumption that the molecular cloud is formed by clumps, which produce spectra within this line-width interval seems to be a natural explanation for the non-thermal population. The observed spectra can be the result of the superposition of individual clump spectra with different central velocities. This model was applied to determine the physical conditions of the observed regions, providing satisfactory results for most of them. However, for some sources the model is not adequate to reproduce the observations, indicating that some other effects should be included, such as IR continuum, that was neglected in the radiative transfer calculations.
\end{abstract}

\section{Introduction}

$\mathrm{NH}_{3}$ emission was observed in in the southern hemisphere both in the direction of dark clouds and giant HII regions (Bourke et al. 1995, Vilas-Boas, \& Abraham 2000). Linewidths in dark clouds are compatible with what is expected from their kinetic temperatures, which are assumed to be equal to the excitation temperatures. However, linewidths in regions associated to star formation are much larger, suggesting the presence of turbulent motions. Another peculiarity of these regions is the existence of asymmetries in the intensity of the $\mathrm{NH}_{3}(\mathrm{~J}, \mathrm{~K})=(1,1)$ hyperfine components, implying departure from LTE conditions. The non-thermal level population in the ammonia molecule was explained by Matsakis et al. (1977) as due to selective trapping in the hyperfine transitions of $\mathrm{NH}_{3}(\mathrm{~J}, \mathrm{~K})=(2,1)-(1,1)$. This interpretation requires linewidths between 0.3 and $0.6 \mathrm{~km} \mathrm{~s}^{-1}$. They can be obtained if the cloud is formed by clumps,

\footnotetext{
${ }^{1}$ Operated by CRAAE, Centro de Radio Astronomia e Aplicações Espaciais, São Paulo, Brazil
} 
each originating a narrow line, the superposition of the spectra of all clumps, with a central velocity distribution, would result in the observed spectrum. This scenario allows the calculation of the physical conditions in each clump from the intensity ratio between the satellites and the main line in the $\mathrm{NH}_{3}(\mathrm{~J}, \mathrm{~K})=(1,1)$ transitions. Stutski \& Winnewisser (1985) solved numerically radiation transfer equations in NLTE conditions, calculated the population of the relevant quantum levels and the relative intensities of the main and hyperfine transitions and applied the results to a few regions. Caproni, Abraham, \& Vilas-Boas (2000) also used their calculations to determine the physical conditions in an extended region around NGC 6334I. In this paper we present $\mathrm{NH}_{3}$ observations towards strong CS(2-1)sources, which have IRAS colors characteristic of compact HII regions. We detected the presence of $\mathrm{NH}_{3}$ in NLTE conditions and applied the diagnostics of Stutzki \& Winnewisser (1985) to determine their physical conditions.

\section{Results}

The observations were made with the $13.6 \mathrm{~m}$ Itapetinga radiotelescope, using a cryogenic radiometer and an acusto-optical spectrometer. They are part of a survey of $\mathrm{NH}_{3}$ emission in the direction of CS sources that have IRAS colors characteristic of ultra-compact HII regions. The $\mathrm{NH}_{3}(\mathrm{~J}, \mathrm{~K})=(1,1)$ transition was detected in 24 sources, 17 spectra show clear signs of NLTE, 4 are compatible with LTE population and 3 have a very low signal to noise ratio.
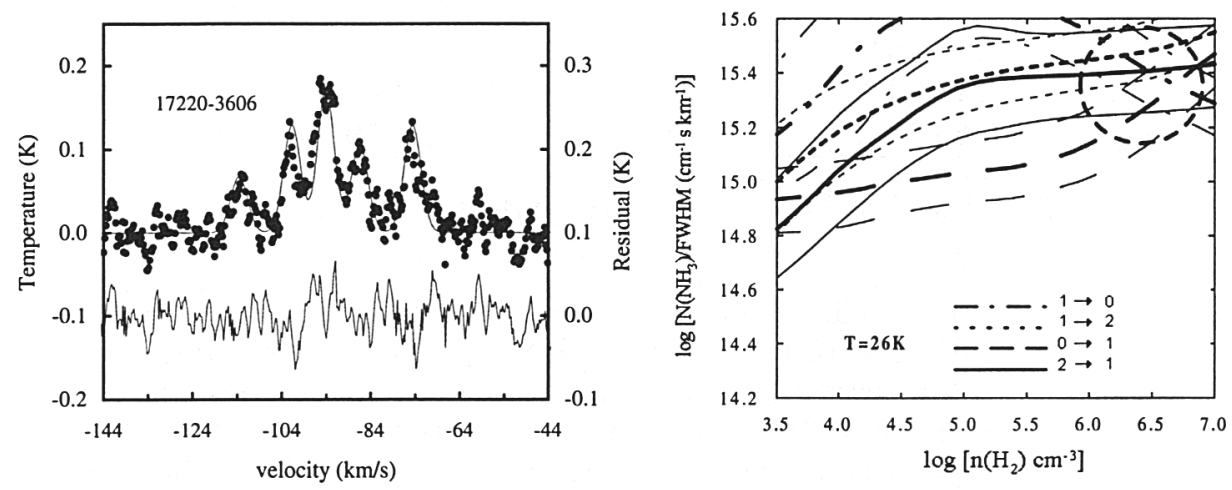

Figure 1. Left: $\mathrm{NH}_{3}$ spectrum of the IRAS source 17220-3606, points are the observations, continuum line gaussian fits. Right: lines representing the ratio of hyperfine to main line intensities. Circle represents the region in which the lines intersect.

Solutions of the NLTE radiation transfer equations given by Stutski \& Winnewisser (1985) were used to determine the ammonia column density $\mathrm{N}\left(\mathrm{NH}_{3}\right)$, molecular hydrogen density $n\left(\mathrm{H}_{2}\right)$ and excitation temperatures $T$. To get these parameters we fist preceded to determine, by fitting gaussians to the spectrum, 


\begin{tabular}{cccc}
\hline Source & $\begin{array}{c}N\left(\mathrm{NH}_{3}\right) \\
10^{14}\end{array}$ & $\begin{array}{c}n\left(\mathrm{H}_{2}\right) \\
\mathrm{cm}^{-3}\end{array}$ & $\begin{array}{c}\mathrm{T} \\
\mathrm{K}\end{array}$ \\
\hline $12320-6122$ & $(2-3)$ & $(2-6) \times 10^{4}$ & 24 \\
& $(4-6)$ & $(2-6) \times 10^{6}$ & 24 \\
$12383-6128$ & - & - & - \\
$13079-6218$ & - & $(3-10) \times 10^{6}$ & - \\
$15394-5358$ & $(1.5-2.5)$ & 15 \\
$15408-5356$ & $(2.5-4.0)$ & $10^{4}-10^{7}$ & 18 \\
$15411-5352$ & $(2-4)$ & $10^{5}-10^{7}$ & 15 \\
$16065-5158$ & $(2-6)$ & $10^{4}-10^{7}$ & 15 \\
$16164-5046$ & $(4-8)$ & $10^{4}-10^{7}$ & 18 \\
$16172-5028$ & $(3-8)$ & $(1-2) \times 10^{4}$ & 19 \\
& $(3-8)$ & $(3-9) \times 10^{6}$ & 19 \\
$16177-5018$ & - & - & - \\
$16351-4722$ & - & - & - \\
$16484-4603$ & - & - & - \\
$16506-4512$ & - & - & - \\
$16547-4247$ & - & - & - \\
$16506-4512$ & - & - & - \\
$17009-4042$ & - & $(3-16) \times 10^{5}$ & 30 \\
$17059-4132$ & $(2-3)$ & - & - \\
$17136-3617$ & - & $4 \times 10^{6}$ & - \\
$17175-3544$ & - & $(1-3) \times 10^{4}$ & 17 \\
$17220-3606$ & 25 & $(3-9) \times 10^{6}$ & 17 \\
$17233-3609$ & $4-10$ & - & - \\
& $4-10$ & - & - \\
$17258-3637$ & - & $(3-9) \times 10^{4}-10^{7}$ & 18 \\
$17271-3439$ & $3-6$ & & \\
$17278-3541$ & $(4-6)$ & - & - \\
\hline & & & - \\
\hline
\end{tabular}

Table 1. Physical Parameters of the $\mathrm{NH}_{3}$ sources.

the flux density of the each hyperfine component relative to the main line flux density. Curves of these ratios in the $\log \left[\mathrm{N}\left(\mathrm{NH}_{3}\right) / \mathrm{FWHM}\right]-\log \left[\mathrm{n}\left(\mathrm{H}_{2}\right)\right]$ obtained by Stutski and Winnewisser (1985) for five temperatures were digitized and interpolated in intervals of $1 \mathrm{~K}$. The observed ratios were plotted in the same graph for all temperatures. The intersection of the four lines gave the ammonia column density and molecular hydrogen density. This intersection is obtained only in the graph where the temperature coincides with the source temperature. An example of this situation is given in Figure 1, where the circle represents the region where the four lines intersect. The parameters obtained for all the observed sources are given in Table 1, where the 24 sources are listed in column 1 , the ammonia column density, molecular hydrogen volume density and kinetic temperature are presented in columns 2, 3 and 4 respectively. For some sources two possible intersections were found, one for low and the other for high $\mathrm{H}_{2}$ density, they are presented in different lines in the Table. On others, the curves overlap over a large interval of $\log \left[n\left(\mathrm{H}_{2}\right)\right]$. This ambiguity can be solved with 
we did not find solutions for any value of $\mathrm{T}$, as shown in Figure 2. It is possible that in these sources some of the assumptions made in the model are not valid, as for example neglecting external radiation.
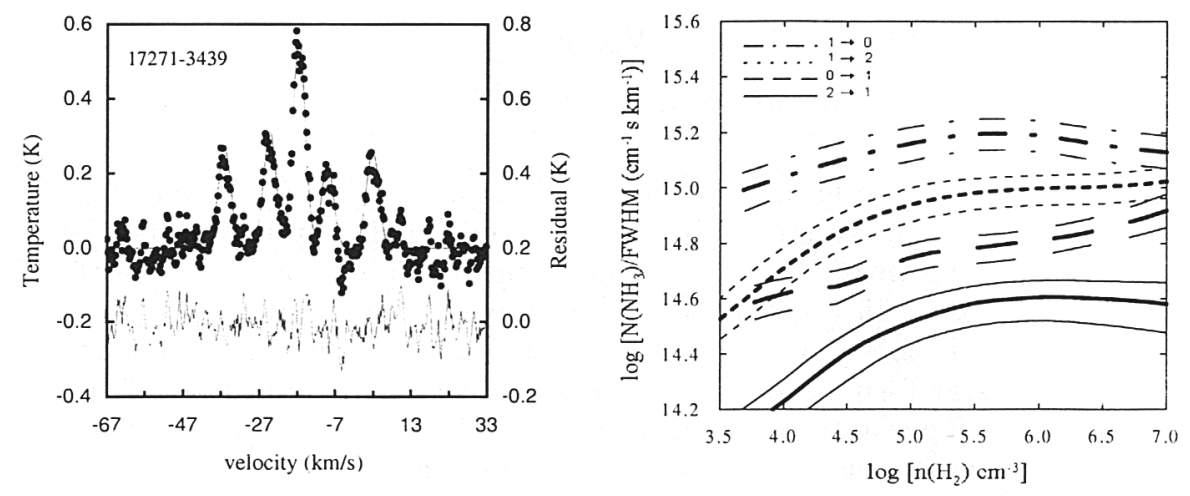

Figure 2. Same as Figure 1 for the source 17271-3439. Crossing does not occur for any temperature.

\section{Conclusions}

We have detected ammonia emission in the direction of CS sources that have IRAS colors characteristic of compact HII regions. The widths of the $\mathrm{NH}_{3}(\mathrm{~J}, \mathrm{~K})$ $=(1,1)$ transitions are much larger than what is expected from kinetic temperatures and the hyperfine intensity ratios show signs of NLTE conditions. The physical parameters of these regions were determined using the numerical solutions of the NLTE level populations given by Stutski \& Winnewisser (1985), assuming that the cloud is formed by dense clumps. Some of the spectra could not be fitted with this simple model, indicating that some other parameter, like and infrared continuum field should be included.

Acknowledgments. This work was partially supported by the Brazilian agencies FAPESP, CNPq and FINEP.

\section{References}

Bourke, T. I., Hyland, A. R., Robinson, G. 1995, MNRAS, 276, 1052B

Caproni, A., Abraham, Z, \& Vilas-Boas, J. W. S. 2000, A\&A, 361, 685

Matsakis, D. N., Brandeshaft, D., Chui, M. F., et al. 1995, ApJ, 214, L67

Stutski, J., \& Winnewisser, G. 1985, A\&A, 144, 13

Vilas-Boas, J. W. S., Abraham, Z. 2000, A\&A, 355, 1115 